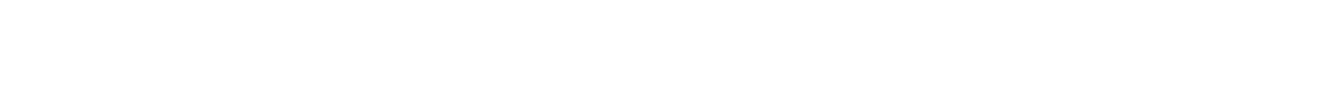

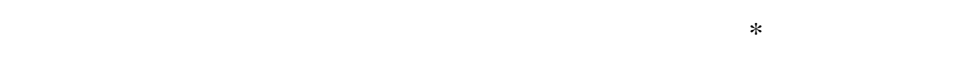

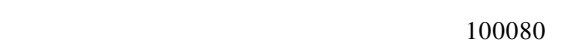

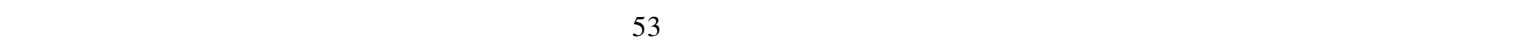

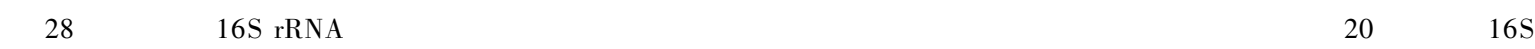

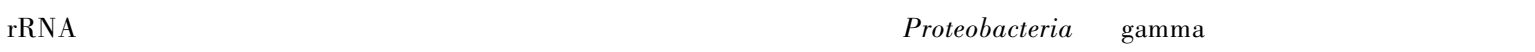

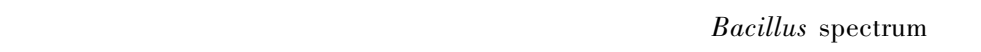

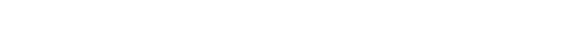

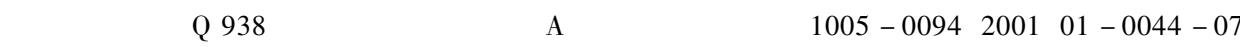

\section{The diversity of Alkaliphiles from Hailaer Soda Lakef-Inner Mongolia}

ZHANG Wei-Zhouf-MAO Wen-Yangf-XUE Yan-Fenf-MA Yan-Hef-ZHOU Pei-Jin Institute of Microbiology $\mathbf{f}$-Chinese Academy of Sciences $\mathbf{f - B e i j i n g ~} 100080$

Abstract£ำifty three strains of organisms were isolated from Hailaer Soda Lakef-nnner Mongolia and DNA was extracted followed by amplification of $16 \mathrm{~S}$ rRNA genes and sequencing. The phenotypic characteristics and the subsequent ARDRA showed that these strains displayed great diversity. Phylogenetic analysis revealed that all of the Gram - negative isolates were confined to the Gamma subdivision of Proteobacteria and that the Gram-positive isolates showed great diversityf-tout most of them belonged to the Bacillus spectrum.

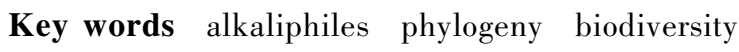

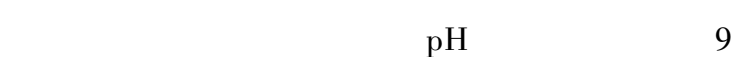

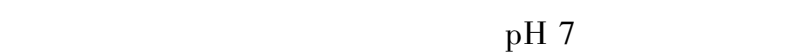

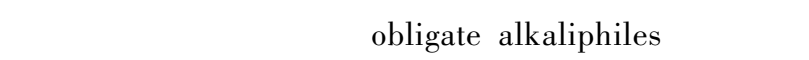
ÊĖ1/

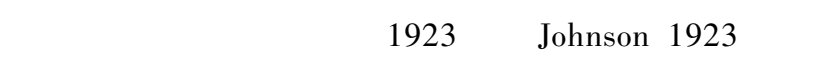

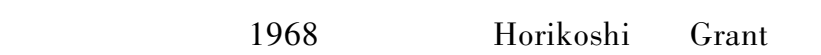

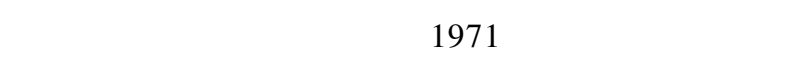

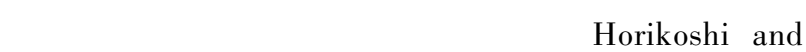

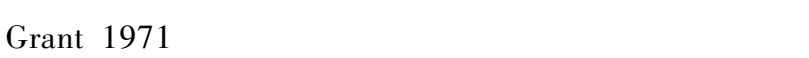

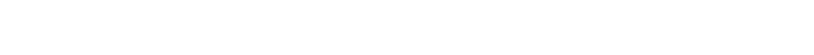

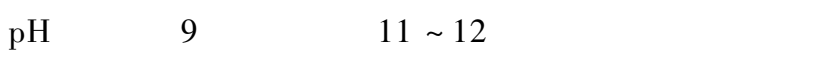

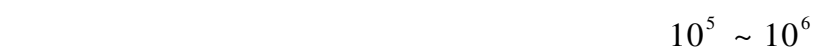

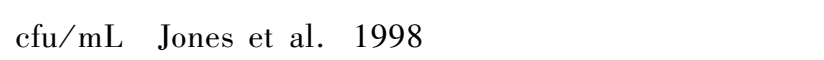

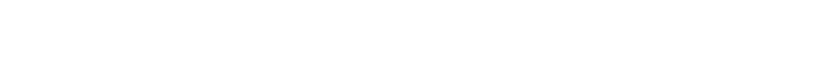

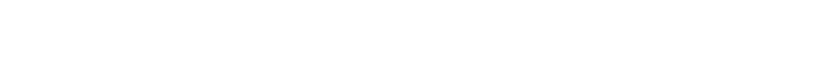

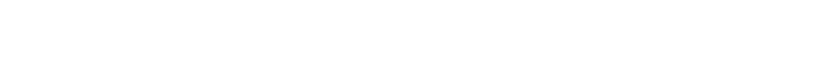

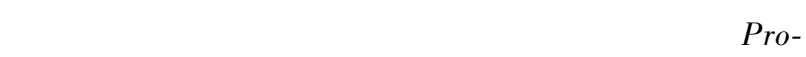

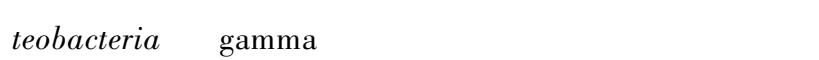

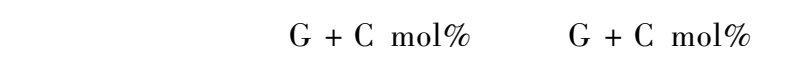
À̀̀j $f$

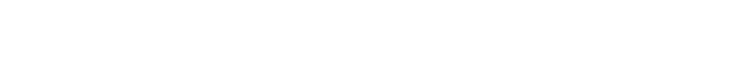

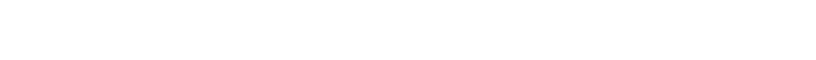

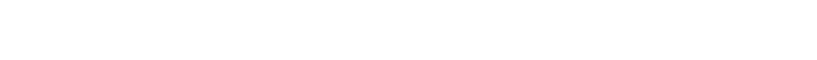

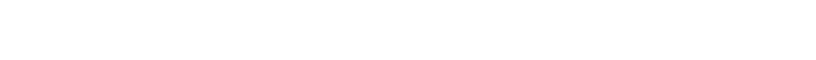

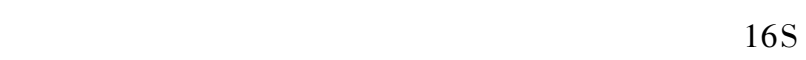

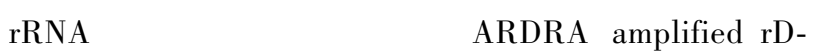
NA restriction analysisf

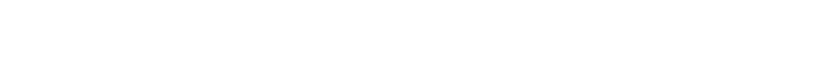

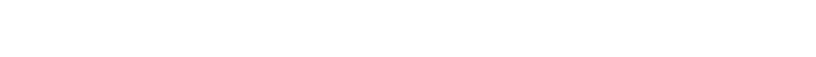
Ï Â»ù' i if

12 ÄÃ ọ́ $\cdot 1 / 2 \cdot$

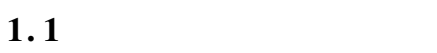




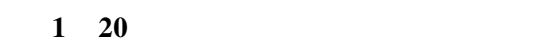

Table 1 Morphological and physiological characteristics of twenty alkaliphilic isolates

\begin{tabular}{|c|c|c|c|c|c|c|c|c|c|c|c|c|c|c|c|c|c|c|c|c|}
\hline Isolate & $\mathrm{Z1}$ & $\mathrm{Z3}$ & $\mathrm{Z} 4$ & $\overline{\mathrm{Z} 6}$ & $\mathrm{Z} 8$ & F1 & F5 & F6 & F8 & F10 & F16 & F23 & F24 & F26 & F27 & $\mathrm{T} 1$ & $\mathrm{~T} 2$ & T5 & T8 & $\mathrm{T} 10$ \\
\hline Morphology & $\mathrm{R}$ & $\mathrm{R}$ & $\mathrm{H}$ & $\mathrm{R}$ & $\mathrm{C}$ & $\mathrm{R}$ & $\mathrm{R}$ & $\mathrm{R}$ & C & $\mathrm{R}$ & $\mathrm{C}$ & $\mathrm{R}$ & $\mathrm{R}$ & $\mathrm{R}$ & $\mathrm{R}$ & $\mathrm{R}$ & $\mathrm{R}$ & $\mathrm{R}$ & C & $\mathrm{R}$ \\
\hline Spore & + & - & - & + & - & - & $\mathrm{N}$ & - & - & + & - & + & - & - & - & + & + & $\mathrm{N}$ & - & + \\
\hline Motility & - & - & + & - & + & $\mathrm{N}$ & + & + & $\mathrm{N}$ & $\mathrm{N}$ & + & - & + & + & + & - & + & + & - & + \\
\hline
\end{tabular}

Growth at NaClf" \% $£(\subset$

$$
0
$$

10

15

$$
20
$$

25

$\%$

0

0.1

0.5

1. 0

1.5

2.0

pH 7.0

Production of

$\mathrm{H}_{2} \mathrm{~S}$

Indole

Catalase

Reduction of

Nitrate

Hydrolysis of

Casein

Starch

Gelatin

Tween 20

Tween 60

Tween 80

Acid from

Glucose

Maltose

Galactose

Sucrose

Fructose

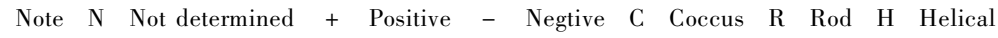

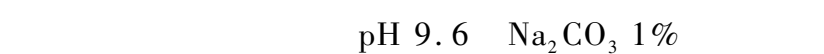

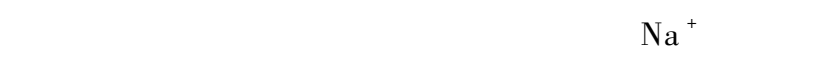

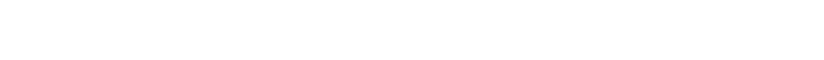

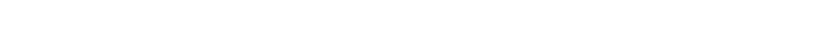

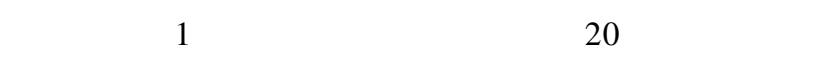

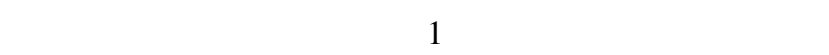

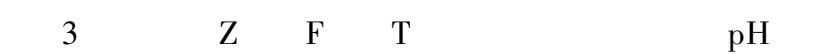

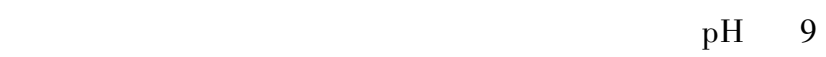

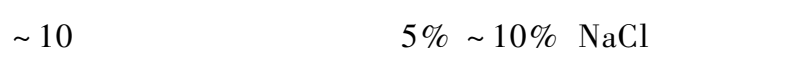

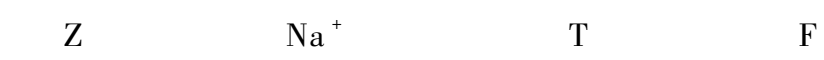

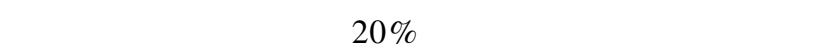

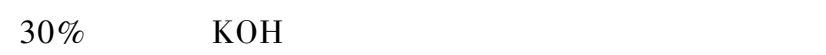

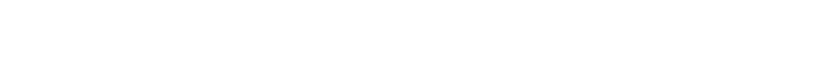

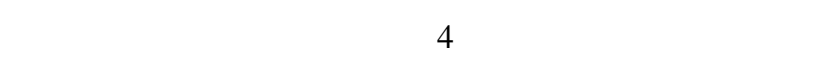

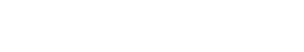

\subsection{ARDRA}

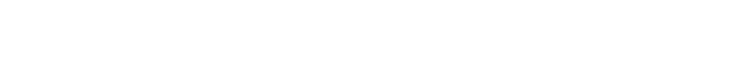

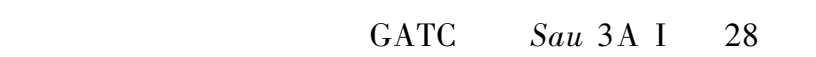

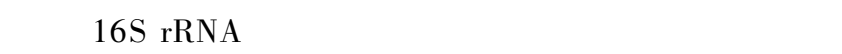

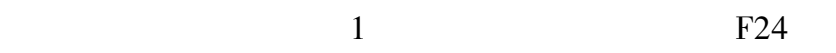

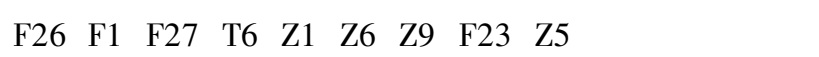

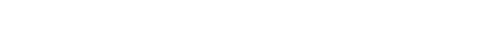

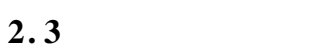

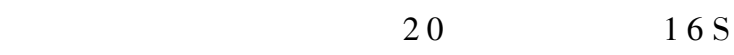




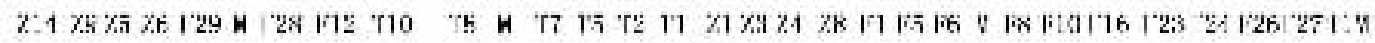
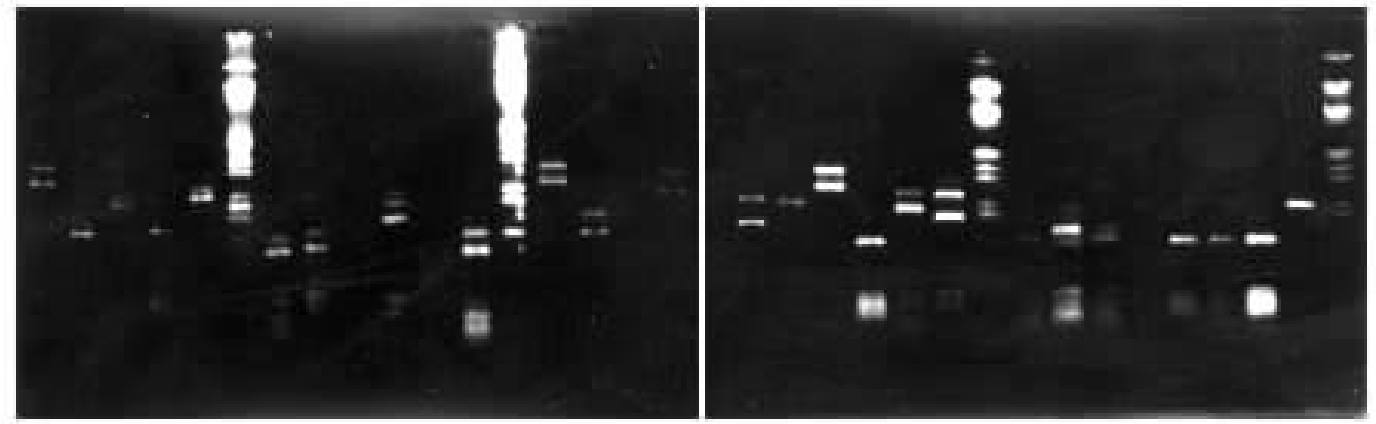

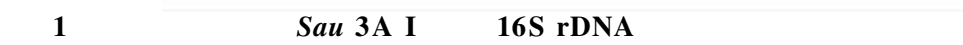

Fig. 1 Agarose gel electrophoresis analysis of restriction of amplified 16S rDNA with Sau 3A I

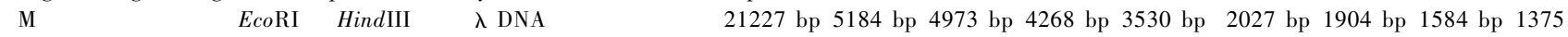
bpi $\$ 47$ bpi $\$ 31$ bpi $\$ 64$ bpi $\$ 25$ bp $£ \subset$

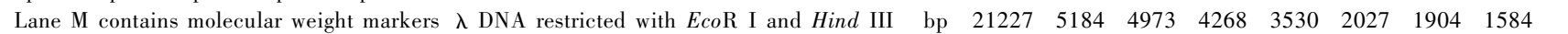
$1375 £-947 £-831 £-564 £-125 £ \subset$

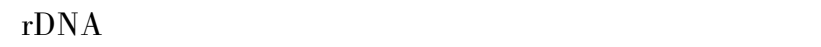

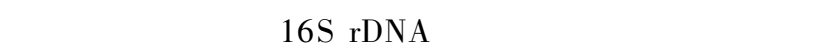

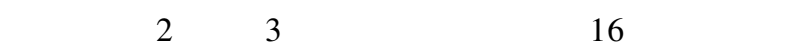

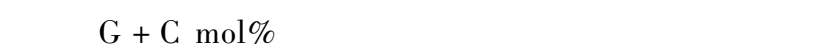

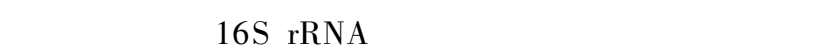

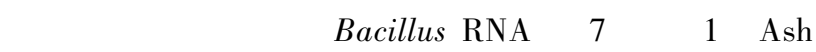

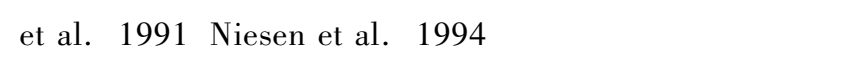

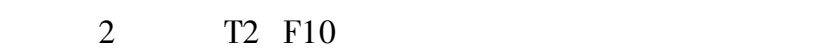
$\mu \ddot{\mathrm{A}}$ Amphibacillusf" Komagata and Shidaf-1997£ध́ Gra-

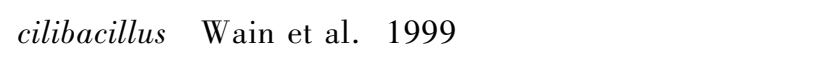
1/低” T2 Ćë Amphibacillus xylanus Ï àË田ôa a $96 \%$ f-F10

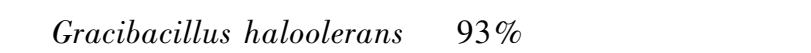

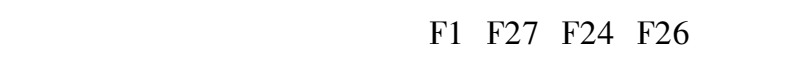

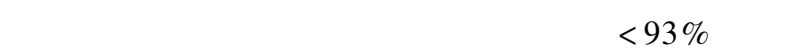

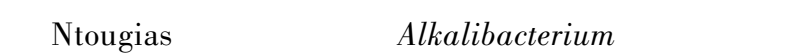

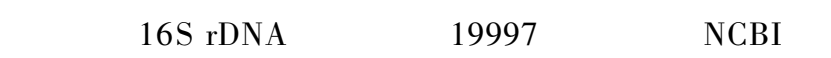

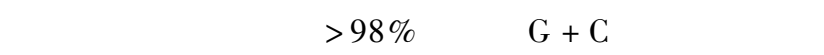

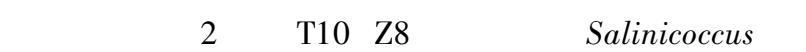
Exiguobacterium fé Salinicoccus roseus of Exiguobacte-

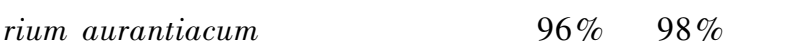

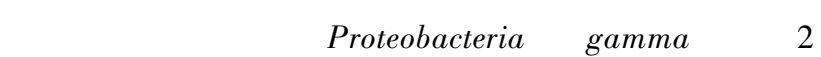

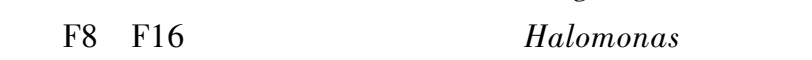

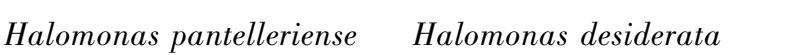

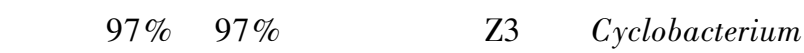

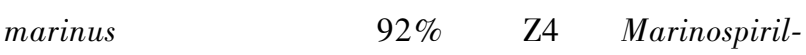
lum minutulum HÄ16S rRNA Í -Ố ĐÔx,

\section{3 İ ÖÂU}

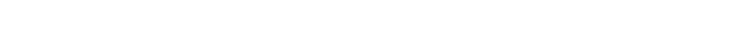

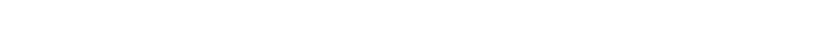

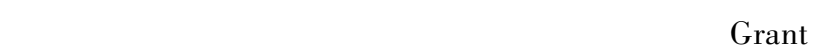
et al. $f+999 £$ Đuchworth et al. $f+996 £ \ngtr$ rant et al. $f \neg$

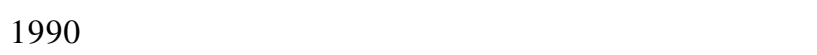

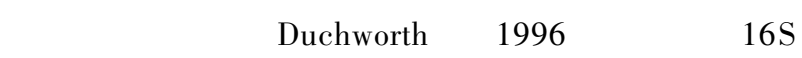

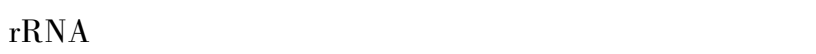

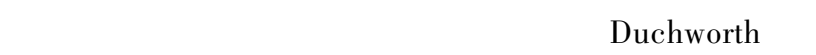

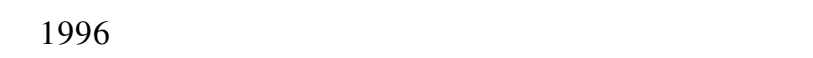

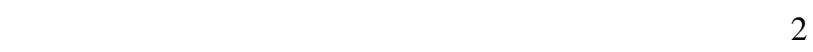

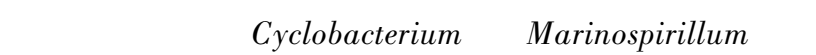
Ốôấ ù

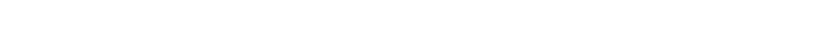

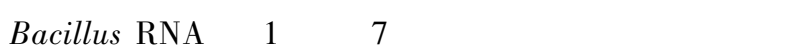

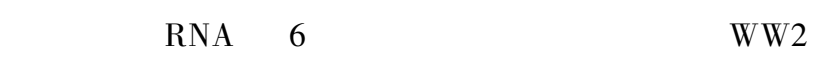

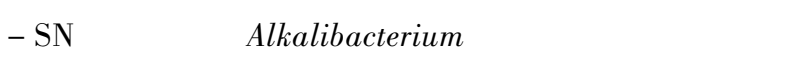

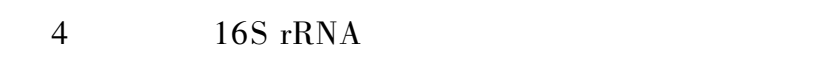

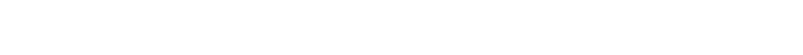

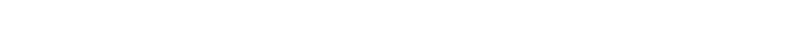

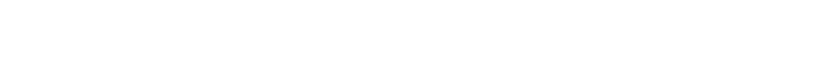

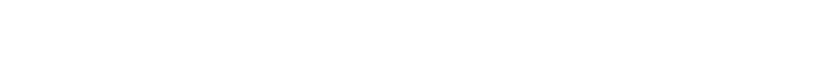

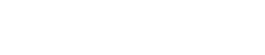

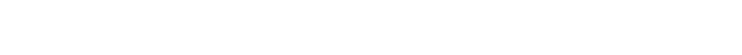

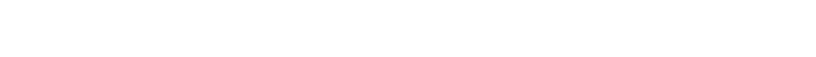

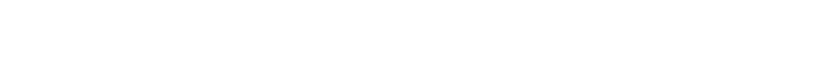

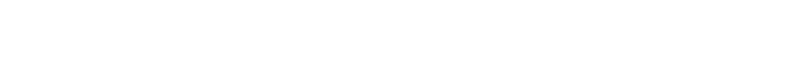

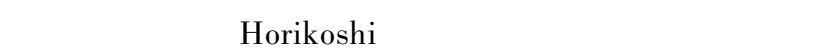

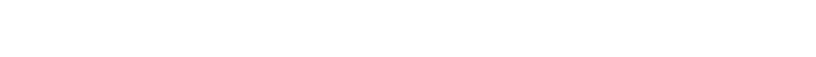

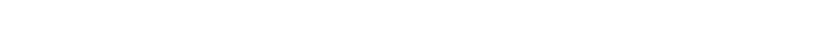




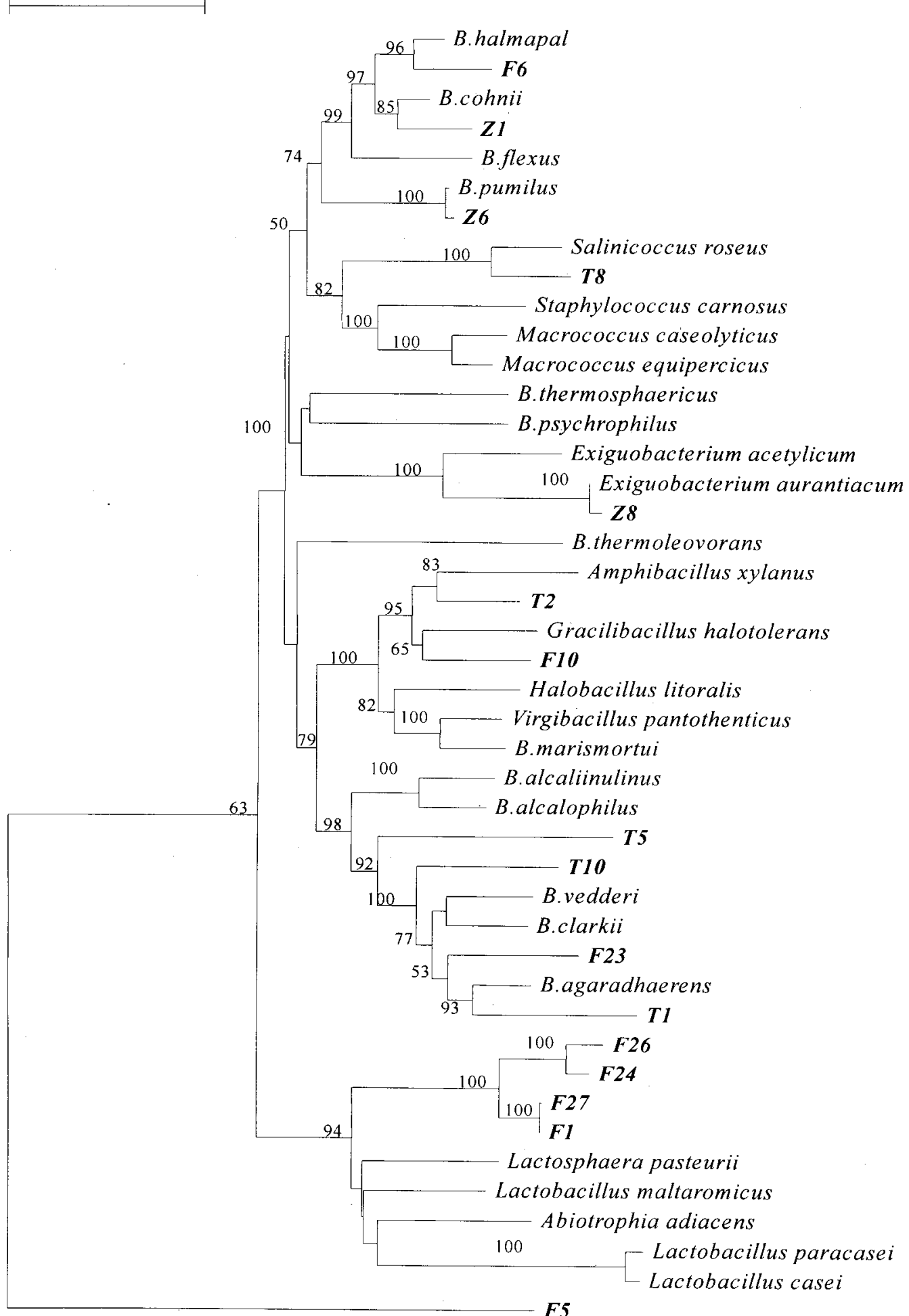

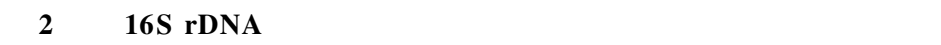

Fig. 2 Phylogenetic tree of the Gram-positive isolates of alkaliphiles based on 16S rDNA sequences

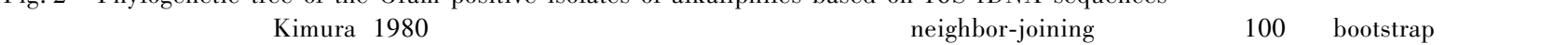

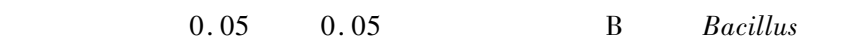

Evolutionary distances were calculated by the method with Kimuraf" 1980 £@wo-parameter calculation model and the topology was inferred by the neighborjoining method based on bootstrap analysis of 100 replicates. Barf $€ .05$ substitutions per nucleotide. B. = Bacillus 
0.1

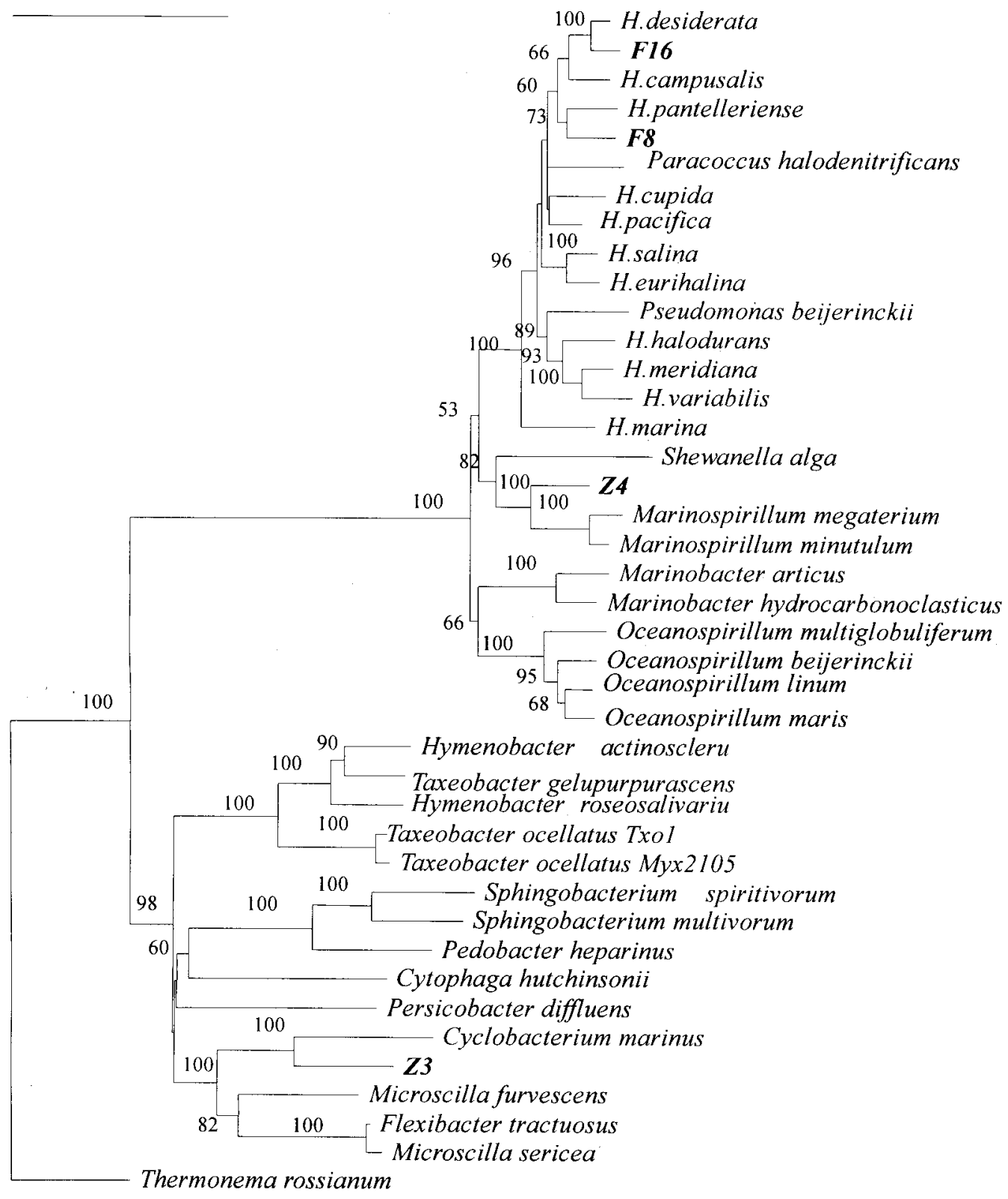

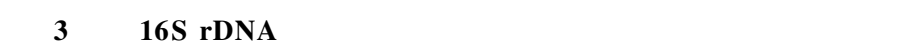

Fig. 3 Phylogenetic tree of the Gram-negative isolates of alkaliphiles based on 16S rDNA sequences

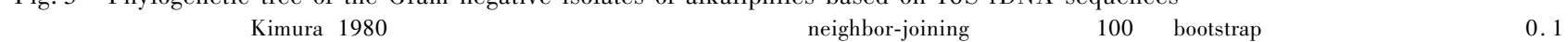

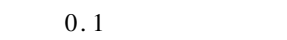

Evolutionary distances were calculated by the method with Kimuraf" $1980 £ @$ wo-parameter calculation model and the topology was inferred by the neighborjoining method based on bootstrap analysis of 100 replicates. Barf€. 1 substitutions per nucleotide. H. = Halomonas

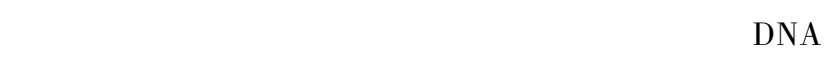

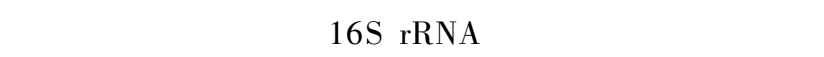

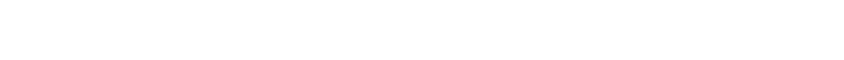

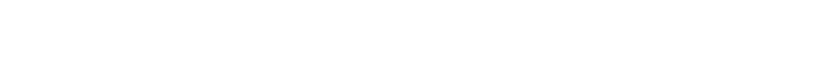

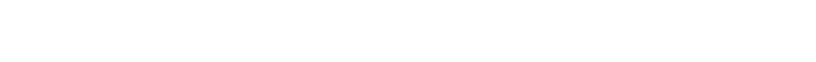

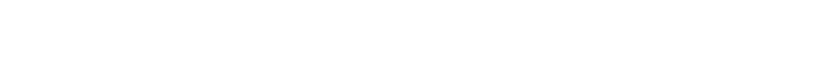

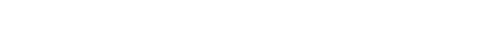

$2 \hat{\imath} i^{1} \hat{h} \ddot{A} \mathrm{I} x$

Ash C, Farrow A E, Wallbanks, Collins M D, 1991. Phylogenetic heterogeneity of the genus Bacillus revealed by comparative analysis of small-subunit-ribosomal RNA sequences. Letter in Applied Microbiology, 13: $202 \sim 206$ 
Duchworth A W, Grant W D, Jones B E, Steenbergen Rvan, 1996. Phylogenetic diversity of soda lake alkaliphiles. FEMS Microbiology Ecology, 19: $181 \sim 191$

Gerhardt P, Murray R G E, Costilow R N, Nester E W, Wood W A, Krieg N R, Phillips G B, 1981. Manual of Methods for General Bacteriology. American Society for Microbiology.

Grant W D, Jones B E, Mwatha W E, 1990. Alkaliphiles: ecology, diversity and applications. FEMS Microbiology Review, 75: $255 \sim 270$

Grant S, Grant W D, Jones B E, Lina Li C K, 1999. Novel archaeal phylotypes from an East African alkaline saltern. Extremophiles, 3: $139 \sim 145$

Gregersen T, 1978. Rapid method for distinction of Gram-negative from Gram-positive bateria. European Journal of Applied Microbiology and Biotechnology, 5: $123 \sim 127$

Horikoshi K, 1971, Production of alkaline enzymes by alkalophilic microorganisms. Part I. Alkaline protease produced by Bacillus No. 221. Agriculture Biological Chemistry, 36: $1407 \sim 1414$

Horikoshi K, Grant W D, 1998. Extremophiles. Ralph Mitchell, Division of Applied Sciences. Harvard University, 6:
$155 \sim 158$

Johnson H W, 1923. Relationships between hydrogen ion, hydroxyl ion and salt concentrations and the growth of seven soil molds. Iowa Agriculture Experiment Stn Research Bulletin, 76: $307 \sim 344$

Jones B E, Grant W D, Duchworth A W, Owenson G G, 1998. Microbial diversity of soda lakes. Extremophiles, 2: 191 200

Marmur J, 1961. A procedure for the isolation of deoxy - ribonucleic acid from microorganisms. Journal of Molecular Biology, 3: $208 \sim 212$

Niesen P, Rainey F A, Onttrup H, Priest F G, 1994. Comparative $16 \mathrm{~S}$ rDNA sequence analysis of some alkaliphilic bacilli and the establishment of a sixth rRNA group within the genus Bacillus. FEMS Microbiology Letters, 117: $61 \sim 66$

Wain M, Tindall B J, Schumann P, Ingrorsen K, 1999. Gracilibacillus gen. nov., with description of Gracilibacillus halotolerans gen. nov, sp. nov., transfer of Bacillus dipaosaurito to Gracilibacillus dipsosaurito comb. nov. and Bacillus salexigens to the genus Salibacillus gen. nov., as Salibacillus salexigens comb. nov. International Journal of Systematic Bacteriology, 49: $821 \sim 831$

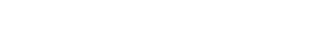

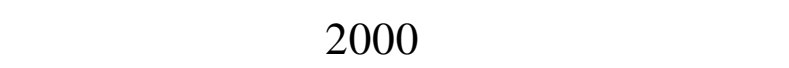

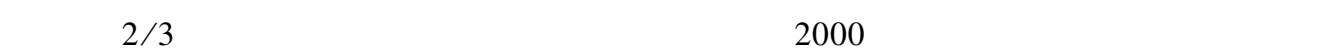

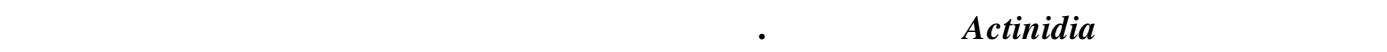

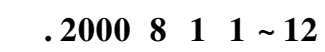

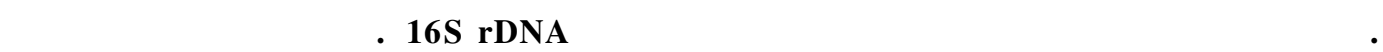
2000 £\&" 2 £④6 $\sim 152$

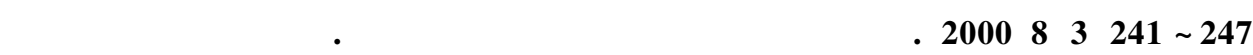

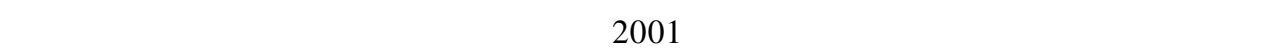

